\title{
onkopediä
}

\section{onkopedia leitlinien}

\section{Periphere T-Zell Lymphome}

\section{Leitlinie}

ICD10: C84.-

Empfehlungen der Fachgesellschaft zur Diagnostik und Therapie hämatologischer und onkologischer Erkrankungen

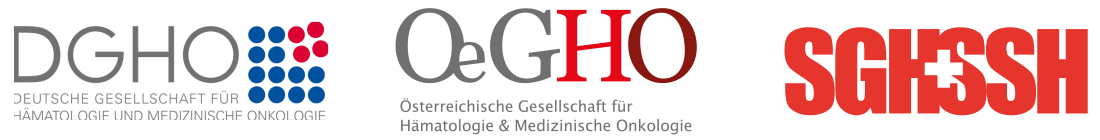




\section{Herausgeber}

DGHO Deutsche Gesellschaft für Hämatologie und Medizinische Onkologie e.V.

Alexanderplatz 1

10178 Berlin

Geschäftsführender Vorsitzender: Prof. Dr. med. Carsten Bokemeyer

Telefon: $+49(0) 3027876089-0$

Telefax: +49(0)3027876089-18

info@dgho.de

www.dgho.de

\section{Ansprechpartner}

Prof. Dr. med. Bernhard Wörmann

Medizinischer Leiter

\section{Quelle}

www.onkopedia.com

Die Empfehlungen der DGHO für die Diagnostik und Therapie hämatologischer und onkologischer Erkrankungen entbinden die verantwortliche Ärztin / den verantwortlichen Arzt nicht davon, notwendige Diagnostik, Indikationen, Kontraindikationen und Dosierungen im Einzelfall zu überprüfen! Die DGHO übernimmt für Empfehlungen keine Gewähr. 


\section{Inhaltsverzeichnis}

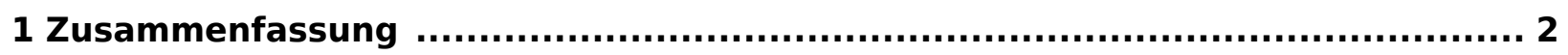

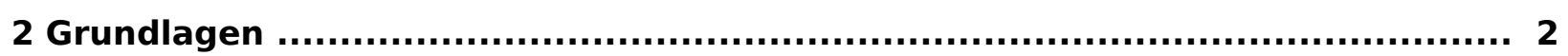

2.1 Definition und Basisinformation .................................................................. 2

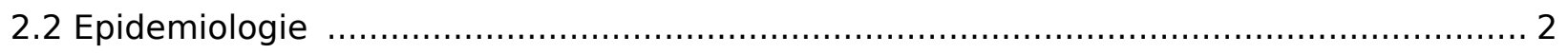

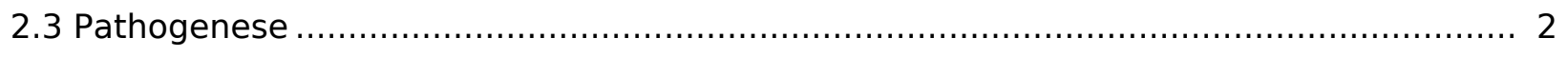

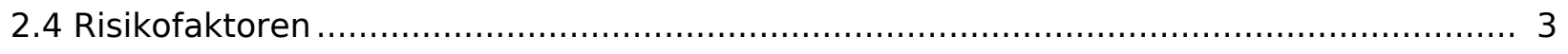

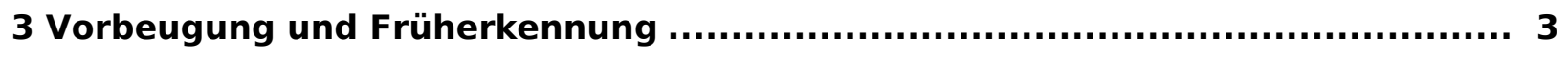

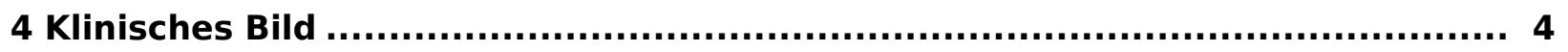

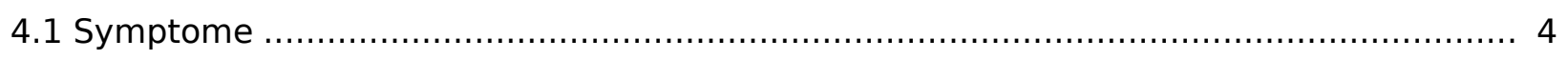

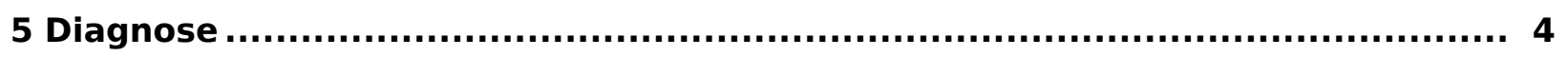

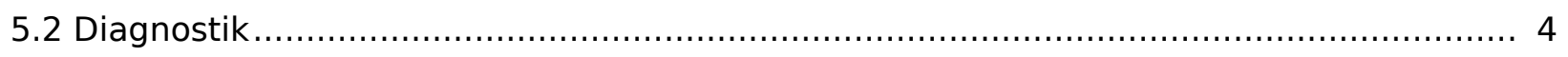

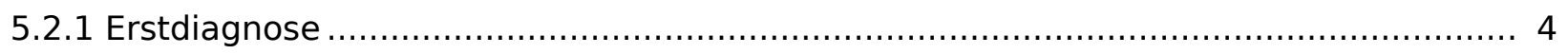

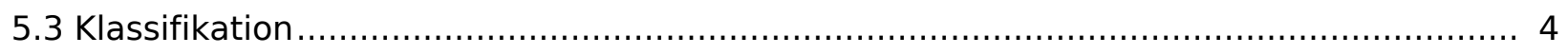

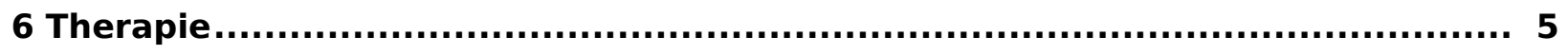

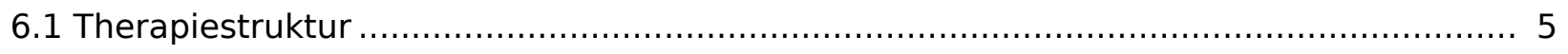

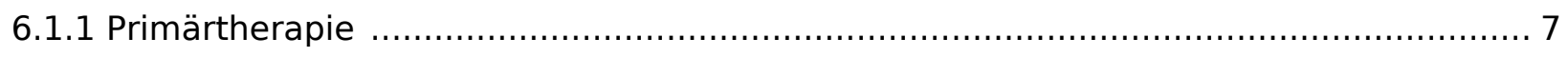

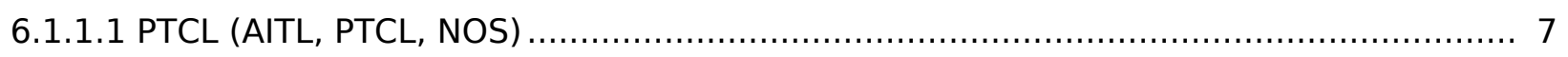

6.1.1.2 Extranodale NK/T-Zell Lymphome vom nasalen Typ ..................................... 7

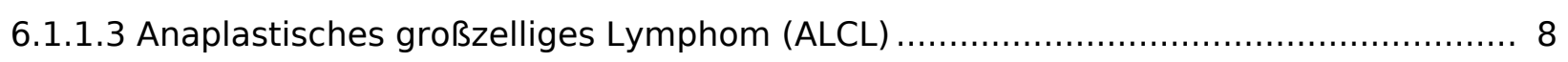

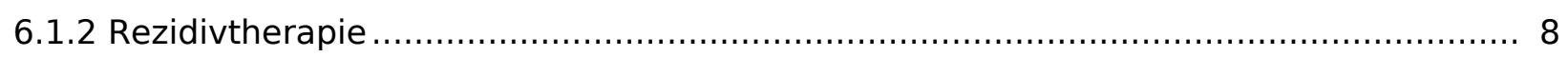

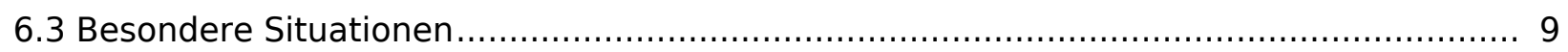

6.3.1 Enteropathie-assoziertes T-Zell Lymphom (EATL) ......................................... 9

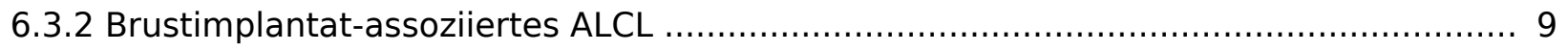

6.3.3 Lymphom-assoziierte Hämophagozytische Lymphohistiozytose ........................ 9

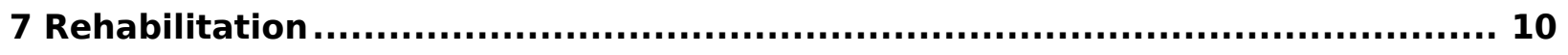

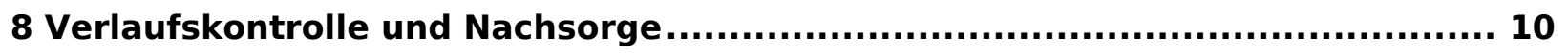

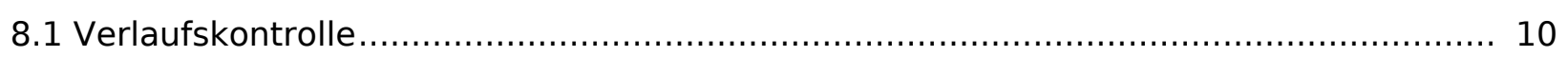

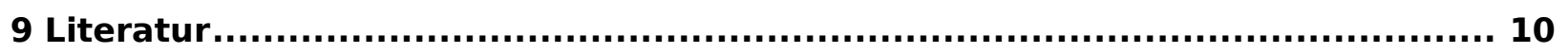

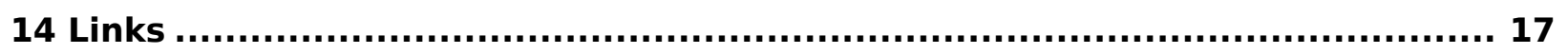

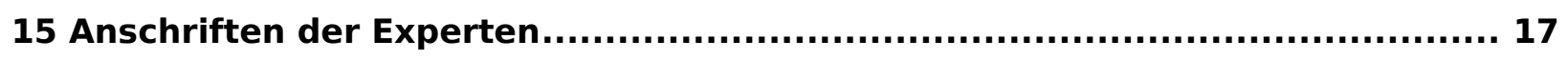

16 Angaben zu möglichen Interessenkonflikten ......................................... 18 


\section{Periphere T-Zell Lymphome}

ICD10: C84.-

Stand: März 2016

Autoren: Georg Hopfinger, Fatime Krasniqi, Peter Reimer, Norbert Schmitz, Gerald Wulf

\section{Zusammenfassung}

Unter dem Begriff der Peripheren T-Zell Lymphome (PTCL) werden klinisch meist aggressiv verlaufende Neoplasien ausgehend von reifen T-Zellen zusammengefasst. PTCL machen weniger als $<10 \%$ aller neu diagnostizierten Non-Hodgkin Lymphome aus. Der Therapieanspruch ist primär kurativ, wobei aktuell Protokolle für Patienten mit PTCL/NOS und AITL, Patienten mit extranodalen NK/T-Zell Lymphomen, sowie Patienten ALCL unterschieden werden.

\section{Grundlagen}

\subsection{Definition und Basisinformation}

Unter dem Begriff periphere T-Zell Lymphome (PTCL) werden die klinisch meist aggressiv verlaufenden nodalen und extranodalen Lymphome mit dem Phänotyp reifer post-thymischer NK- und T-Zellen zusammengefasst. Nach der WHO-Klassifikation von 2008 sind sie von unreifen T-Zell Neoplasien (lymphoblastisches T-NHL, T-ALL), sowie den primär kutanen (CTCL, Mycosis fungoides u.a.) und den primär leukämisch verlaufenden reifen T/NK-Zell Neoplasien (T-PLL, T-LGL) abzugrenzen [1]. Innerhalb der PTCL können histologische Subtypen mit nach Lebensalter und Geographie wechselnder Inzidenz unterschieden werden, wobei in Europa das PTCL ohne weitere Spezifizierung (PTCL, NOS), das angioimmunoblastische T-Zell Lymphom (AITL), sowie die großzelligen anaplastischen Lymphome (ALCL) die häufigsten Erscheinungsformen darstellen [2, 3, 4], siehe auch Tabelle 1.

\subsection{Epidemiologie}

PTCL machen weltweit $<10 \%$ aller neu diagnostizierten NHL aus [6] und stellen in Europa und Nordamerika mit Inzidenzen von jeweils $<1$ in 100000 Menschen pro Jahr sehr seltene Lymphome dar $[2,4,6,7]$. Männer sind häufiger betroffen als Frauen $(1,7: 1)$, die Erkrankungswahrscheinlichkeit nimmt - mit Ausnahme des ALCL - mit dem Lebensalter zu [6]. Die Inzidenz der extranodalen NK/T-Zell Lymphome vom nasalen Typ ist hingegen im asiatischen Raum am höchsten [4, 6].

\subsection{Pathogenese}

Diagnostisch relevant ist der Nachweis unterschiedlicher Mutationen, die eine bessere Unterscheidung von Subentitäten ermöglichen können. Bei AITL wurden neben "loss of function“Mutationen von TP53, kETV6, CCND3, und EP300 "gain of-function“-Mutationen in JAK2 und STAT3 identifiziert [8]. Bei PTCL mit follikulären T-Helfer-Typ und AITL, nicht jedoch bei ALCL, kann eine TET2 Mutation nachgewiesen werden [9, 10, 11, 12]. Auf funktioneller Ebene ist TET2 an der Transkription durch DNA-Methylierung beteiligt, weswegen hypomethylierende Substanzen bei PTCL mit TET2-Mutationen wirksam sein könnten. Ferner ist eine Unterscheidung in bis zu 98\% mittels Microarray-Technik zwischen AITL, ALK-negativem ALCL und PTCL, 
NOS möglich, was bei der Erstellung einer korrekten Diagnose hilfreich sein kann [13]. Auch bei der prognostischen Einschätzung kommt der Molekularbiologie eine bedeutende Rolle zu, wobei der Nachweis der reziproken Translokationen des Anaplastic Lymphoma Kinase (ALK) Gens auf 2p23 mit dem Fusionspartner Nucleophosmin (NPM) auf Chromosom 5 ist besonders relevant ist. Die resultierende Translokation $\mathrm{t}(2 ; 5)(\mathrm{p} 23 ; \mathrm{q} 35)$ bzw. das NPM-ALK Fusionsgen können mittels PCR und/oder spezifischen Antikörpern nachgewiesen werden und ermögliche die Unterscheidung in die prognostisch unterschiedlichen Subentitäten ALK-positives und ALK-negatives ALCL [14]. Desweiteren werden bei ALK-negativem ALCL in ca. 30\% DUSP22 Mutationen gefunden, die mit einer günstigeren Prognose vergesellschaftet sind [15].

Aus klinischer Sicht interessant ist der Nachweis des Transmembranrezeptor CD30, welcher auf aktivierten T- und B-Zellen exprimiert wird. Eine starke CD30-Expression ist typischerweise beim Hodgkin-Lymphom und dem ALCL nachweisbar, weswegen der gegen CD30 gerichtete Antikörper Brentuximab-Vedotin hier besonders wirksam ist und als Rezidivtherapie zugelassen ist. Bei anderen PTCL Subtypen ist die CD30-Expression in der Immunhistochemie (ICH) [16] oder Durchflusszytometrie [17] sehr heterogen. Interessanterweise ist jedoch die Stärke der CD30-Expression bei PTCL nicht eindeutig mit dem Ansprechen auf Brentuximab-Vedotin korreliert und auch bei CD30 negativen Fälle wurde ein Ansprechen beobachtet [18]. Möglicherweise können durch Kombination von $\mathrm{ICH}$ und Nachweis von CD30 mRNA die Detektionssicherheit verbessert werden [19].

\subsection{Risikofaktoren}

Eine längerfristige Immunsuppression ist generell mit einem vermehrten Auftreten von NonHodgkin-Lymphomen einschließlich der PTCL verbunden [6]. Ein Beispiel ist das hepatosplenische T-Zell Lymphom, das in ca. $20 \%$ der Fälle bei Patienten mit vorbestehender Immunsuppression gefunden wird [20]. Für das Auftreten eines Enteropathie-assoziierten T-Zell Lymphoms (EATL) stellt eine vorbestehende Zöliakie einen Risikofaktor dar, wobei der mit Zöliakie in 80-90 \% assoziierte EATL Typ 1 typischerweise CD56-negativ sowie eine Deletion des langen Arms von Chromosom 16q- und Zugewinn an Chromosom 9q zu finden ist. Beim CD56-positiven EATL Typ 2 besteht keine Assoziation mit der Zöliakie [21, 22]. In genomweiten Assoziationsstudien wurden bisher keine etwaigen immungenetischen Mechanismen beschrieben, eine Ausnahme stellt der Polymorphismus im TNF-Gen mit erhöhter Suszeptibilität für das Auftreten von Mycosis fungoides dar [23]. Patienten mit ATLL sind typischerweise HTLV-I seropositiv, weswegen die Prävention der vertikalen Übertragung von HTLV-I die Lymphom-Inzidenz senken könnte [24]. Im Gegensatz dazu ist der pathogenetische Mechanismus einer Assoziation von EBV-Reaktivierung und dem Auftreten verschiedenen Lymphome wie z.B. NK/T-Zell Lymphomen unklar [25].

\section{Vorbeugung und Früherkennung}

Spezifische Empfehlungen zur Vorbeugung oder Früherkennung können aus den epidemiologischen Daten nicht abgeleitet werden. 


\section{Klinisches Bild}

\subsection{Symptome}

Wie bei den aggressiven B-Zell Lymphomen treten als Initialmanifestation zumeist progrediente schmerzlose Lymphknotenvergrößerungen und/oder extranodale Manifestationen, bei den EATL insbesondere im Gastrointestinaltrakt, bei den extranodalen NK/T-Zell Lymphomen im HNO-Bereich, sowie bei den Panniculitis-like T-Zell Lymphomen als subkutane Indurationen auf. Eine B-Symptomatik ist typisch. Eine Infektneigung, z.T. mit opportunistischen Erregern findet sich gehäuft [26]. Bei Patienten mit AITL liegen häufig charakteristische, serologische Befunde vor, die als dysregulierte humorale Immunreaktion interpretiert werden, wie $z$. B. polyklonale Hypergammaglobulinämie oder Coombs-positive hämolytische Anämie [27].

\section{Diagnose}

\subsection{Diagnostik}

\subsubsection{Erstdiagnose}

Aufgrund ihres seltenen Auftretens und der ausgeprägten histologischen Heterogenität ist eine eingehende immunhistopathologische Diagnostik mit referenzpathologischer Beurteilung für die Therapiesteuerung unumgänglich [28]. Oftmals ist der Klonalitätsnachweis der pathologischen T-Zellen hilfreich. Daneben wurden eine Reihe von immunologischen und molekularen Markern zur Sicherung der Diagnose und Festlegung des Subtyps etabliert $[2,29]$. Bei V.a. das Vorliegen eines ALCL ist der Nachweis der Expression von CD30 sowie die Bestimmung des Mutationsstatus für die Translokation $\mathrm{t}(2 ; 5)$ mit Detektion des NPM-ALK Fusionsgens essentiell. Bei pädiatrischen Patienten konnte gezeigt werden, dass neben der Suche nach NPM-ALK Transkripten im Blut oder Knochenmark (RT-PCR) die Bestimmung von ALK Antikörpertitern in Plasma einen hohen prognostischen Wert besitzen [30]. Die Ausbreitungsdiagnostik und Stadieneinteilung der T-Zell Lymphome erfolgt analog zu aggressiven BZell Lymphomen, siehe Onkopedia Diffuses Großzelliges B-Zell-Lymphom. Eine Knochenmarkdiagnostik (Aspiration und Biopsie) inkl. Durchflusszytometrie zur Immunphänotypisierung ist obligat. An bildgebender Diagnostik sind eine CT von Hals, Thorax und Abdomen erforderlich. Eine CT oder MRT-Untersuchung des Schädels oder eine Lumbalpunktion werden nur bei klinischem Verdacht empfohlen. Das PET besitzt bei extranodalen nicht-kutanen Manifestationen, insbesondere im Gl-Trakt, eine der CT überlegene Sensitivität. So können beim Staging von PTCL mittels FDG-PET häufig zusätzliche Manifestationen gefunden werden [31, 32]. Für eine Risikostratifizierung stehen mehrere Möglichkeiten zu Verfügung, wobei in der klinischen Praxis der für aggressive B-Zell Lymphome entwickelte Internationale Prognostische Index (IPI) in der Regel ausreicht. Alter über 60 Jahre, eine erhöhte LDH, ein Performance-Status 3-4, Stadium $\geq \mathrm{III}$ und mehr als ein extranodaler Befall gehen in die Abschätzung des individuellen Risikos ein [33]. Es stehen auch weitere Risikoscores wie z.B. der "Prognostic Index for T-Cell Lymphoma" (PIT) [34] zur Verfügung, wobei eine Überlegenheit gegenüber dem Standard-IPI nicht klar belegt ist.

\subsection{Klassifikation}

Die PTCL entsprechend der WHO Klassifikation sind in Tabelle 1 dargestellt. 
Tabelle 1: PTCL und NK-Neoplasien nach WHO-Klassifikation 2008

\begin{tabular}{|c|c|}
\hline Bezeichnung & Abkürzung \\
\hline T-Prolymphozyten Leukämie & T-PLL \\
\hline T-Zell große granuläre Lymphozytenleukämie & T-LGL \\
\hline \multicolumn{2}{|l|}{ Chronische lymphoproliferative Störungen der NK-Zellen } \\
\hline \multicolumn{2}{|l|}{ Aggressive NK-Zell Leukämie } \\
\hline \multicolumn{2}{|c|}{$\begin{array}{l}\text { EBV-positive T-Zell lymphoproliferative Störungen in der Kindheit } \\
\text { Systemische EBV-positive T-Zell lymphoproliferative Erkrankung im Kindesalter } \\
\text { Hydroavacciniformia-like Lymphom }\end{array}$} \\
\hline Adulte(s) T-Zell Leukämie/Lymphom & ATLL \\
\hline Extranodales NK/T-Zell Lymphom, nasaler Typ & $\mathrm{NT} / \mathrm{TCL}$ \\
\hline Enteropathie-assoziiertes T-Zell Lymphom & EATL \\
\hline Hepatosplenisches T-Zell Lymphom & HSTL \\
\hline Subkutanes panniculitis-like T-Zell -Lymphom & SPTCL \\
\hline Mycosis fungoides & MF \\
\hline Sézary Syndrom & SS \\
\hline $\begin{array}{l}\text { Primär kutane CD30-positive T-Zell lymphoproliferative E } \\
\text { Primär kutanes anaplastisches großzelliges Lymphom } \\
\text { Lymphomatoide Papulose }\end{array}$ & C-ALCL \\
\hline \multicolumn{2}{|c|}{$\begin{array}{l}\text { Primär kutane periphere T-Zell Lymphome, seltene Subtypen } \\
\text { Primär kutanes gamma/delta T-Zell Lymphom } \\
\text { Primär kutanes CD8+ aggressives epidermotropes zytotoxisches T-Zell Lymphom } \\
\text { Primär kutanes CD4+ klein/mittelgroßzelliges T-Zell Lymphom }\end{array}$} \\
\hline Peripheres T-Zell Lymphom, nicht weiter spezifiziert & PTCL, NOS \\
\hline Angioimmunoblastisches T-Zell Lymphom & AITL \\
\hline Anaplastisch großzelliges Lymphom (ALCL), ALK-positiv & ALCL, ALK+ \\
\hline Anaplastisch großzelliges Lymphom (ALCL), ALK-negativ & ALCL, ALK- \\
\hline
\end{tabular}

\section{Therapie}

\subsection{Therapiestruktur}

Die PTCL sind als aggressive Lymphome ohne Behandlung rasch progredient, indolente Verlaufsformen des AITL stellen Ausnahmen dar [35]. Mittels systemischer Chemotherapie sind die PTCL bei einem Teil der Patienten heilbar. Für Patienten, bei denen aufgrund eingeschränkter Verträglichkeit eine volldosierte Systemtherapie nicht durchgeführt werden kann, können angepasste Therapien in palliativer Intention sinnvoll sein.

Ein Therapiealgorithmus, stratifiziert nach Histologie, Therapieverträglichkeit, sowie Erkrankungssituation (Erstlinientherapie, Zweitlinientherapie) ist in Abbildung 1 zusammengefasst. 

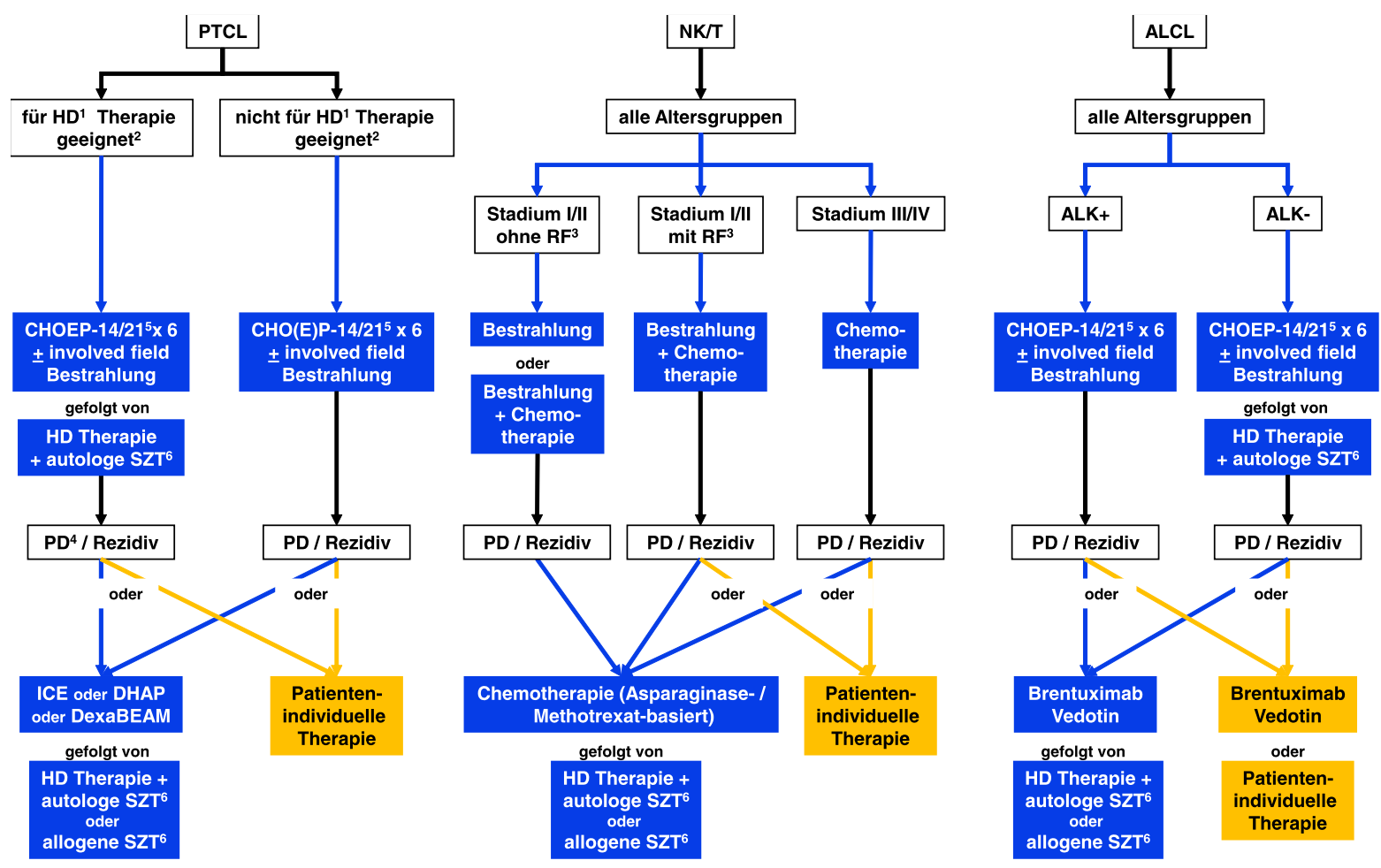

Legende:

Kurative Therapie; __ palliative Therapie;

${ }^{1}$ HD: bei primärer Chemosensitivität (CR1 oder PR1) autologe Stammzelltransplantation (ASZT) als Option;

2 Therapie-Eignung entsprechend lokaler Richtlinien;

${ }^{3}$ NK/T Risiko Score [44] Low-risk: Ann Arbor l, keine Tumorinvasion $\leq 60$ Jahre, keine erhöhte LDH, ECOG PS: 0-1;

${ }^{4}$ bei PD oder primär refraktärer Erkrankung wird bei Patienten die für eine Hochdosistherapie geeignet erscheinen, eine intensive Chemotherapie empfohlen. Bei Patienten die für keine Hochdosistherapie geeignet erscheinen, soll eine individuelle Therapie erwogen werden, da dazu keine ausreichenden Daten für eine Empfehlung existieren.

5 Für Patienten die für eine HD als geeignet eingestuft wurden, wird als primäre Therapie CHOEP (CHOP+Etoposid)14 oder CHOEP12 empfohlen. Für Patienten die für eine HD als nicht geeignet eingestuft wurden, wird eine primäre Therapie mit CHOP14 oder CHOP21 empfohlen, in individuellen Fällen kann auch CHOEP 14 oder CHOEP 12 erwogen werden.

${ }^{6}$ Als HD Therapie in erster Remission( CR1 od PR1) wird eine ASCT empfohlen, für eine allogene SZT gibt es derzeit keine unterstützenden Daten. Eine HD in CR2 oder PR2 kann mit ASZT oder allogener SZT durchgeführt werden

Die Therapie des extranodalen NK/T-Zell Lymphomen, der ATLL und des ALCL unterscheidet sich grundsätzlich von der Therapie des PTCL, NOS und des AITL. Andere T-Zell Lymphome wie das hepatosplenische T-Zell Lymphom bedürfen auf Grund ihres äußerst ungünstigen Verlaufs einer besonders aggressive Therapie, wobei hier intensive Induktionsregime wie ICE (Ifosfamid, Carboplatin, Etoposid) oder IVAC (Ifosfamid, Etoposid, Cytarabin) [36] oder ESHAP (Etoposid, Methylprednisolon, Cytarabin und Cisplatin) gefolgt von autologer Stammzelltransplantation (ASCT) [37] besonders wirksam erscheinen. Wegen der bislang sehr spärlichen Datenlage und der unbefriedigenden Behandlungsergebnisse ist eine Therapie von Patienten mit PTCL innerhalb von Studien zu empfehlen.Der Unterschied zwischen der klassischen Haarzell-Leukämie und der Variante liegt vor allem im Ansprechen auf die Therapie und in der Prognose. Die Klassifikation ist nicht prädiktiv für die Standard-Erstlinientherapie. 


\subsubsection{Primärtherapie}

\subsubsection{PTCL (AITL, PTCL, NOS)}

Für die häufigsten Entitäten wie PTCL, NOS und AITL liegen zahlreiche Daten zur Behandlung mit CHOP-basierten Schemata vor; allerdings fehlen vergleichende prospektive Studien mit CHOP und CHOP-verwandten Chemotherapieprotokollen. Insgesamt sind Stadien- bzw. IPIabhängig 5-Jahres Gesamtüberlebensraten von lediglich 30-50 \% zu erwarten [33]. Basierend auf Phase II Studien und retrospektiven Analysen wird für geeignete Patienten zumeist CHOP ergänzt um Etoposid (CHOEP) in der Primärtherapie eingesetzt [38, 39].

Als besonderes Problem erscheint eine unter Initialtherapien bis zu 40\% primär progrediente Lymphomerkrankung. Für Patienten, die jedoch auf die Primärtherapie gut ansprechen (CR bzw. gute PR), wird eine konsolidierenden Hochdosistherapie mit autologer Stammzelltransplantation (ASCT) empfohlen, da in mehreren Phase II Studien ein Gesamtüberleben nach 5 Jahren von ca. 50\% gezeigt werden konnte [40]. Die beiden größten Studien zur autologen Transplantation scheinen die guten Ergebnisse einer konsolidierenden ASZT zu bestätigen. In der deutschen Studie wurden 83 Patienten zunächst mit 6 Zyklen CHOP behandelt, 71\% der Patienten sprachen an (CR oder PR), 66\% der Patienten konnten der geplanten ASZT zugeführt werden. Nach Mobilisierung entweder mit Dexa-BEAM (Dexamethason, BCNU, Etoposid, Cytarabin und Melphalan) oder ESHAP und Konditionierung mit hochdosiertem Cyclophosphamid und Ganzkörperbestrahlung wurde ein 3-Jahres DFS von 53\% und 3-Jahres OS von 48\% beobachtet [41]. In einer Studie der Nordic Lymphoma Group wurden 166 Patienten mit 6 Zyklen CHOEP-14 behandelt, wobei Patienten über 60 Jahre CHOP ohne Etoposid erhielten. Die ORR für die ganze Kohorte lag bei $82 \%$. Eine Subgruppenanalysen zeigte besonders bei Patienten mit ALK-negativem ALCL ein besseres 5-Jahres OS von 70\% bzw. ein PFS von $61 \%$ verglichen mit AILT (OS 52\%, PFS 49\%) und PTCL, NOS (OS 47\%, PFS 38\%) [42].

Zusammenfassend ist für geeignete Patienten eine Hochdosistherapie nach Ansprechen auf die Primärtherapie (mindestens PR) zu empfehlen. Prospektive Studien zum Stellenwert der Transplantation als Teil der Primärtherapie liegen allerdings nicht vor.

Ob die Erweiterung der Initialtherapie mit $\mathrm{CHO}$ (E)P um weitere Substanzen, z. B. Alemtuzumab, Romidepsin oder Pralatrexat zu einer Steigerung der primären Ansprechrate führt, wird derzeit in mehreren Phase III Studien getestet. Der Einsatz dieser oder anderer Substanzen in der Primärtherapie außerhalb klinischer Studien ist aktuell nicht gerechtfertigt. Die Ergebnisse einer Interimanalyse der AATT-Studie, die randomisiert den Stellenwert einer konsolidierenden autologen bzw. allogenen Stammzelltransplantation (allo SZT) geprüft hat, wurden kürzlich präsentiert [43]. Da jedoch kein Benefit für eine alloSZT in erster CR gezeigt werden konnte, sollte diese Patienten mit rezidivierter oder refraktärer Erkrankung vorbehalten bleiben bzw. innerhalb klinischer Studien erfolgen.

\subsubsection{Extranodale NK/T-Zell Lymphome vom nasalen Typ}

Erfahrungen in der Therapie von extranodalen NK/T-Zell Lymphomen vom nasalen Typ liegen besonders aus asiatischen Registeranalysen und Studien vor. In den lokal begrenzten Stadien I und II ist die Bestrahlung das zentrale Modul der Therapie mit kurativem Anspruch. Im Stadium I ohne Risikofaktoren scheint die alleinige Strahlentherapie ausreichend zu sein [44], während im Stadium I mit Risikofaktoren (Alter $>60$ Jahre, regionale LK, lokale Tumorinfiltration, erhöhte $L D H$, hohe Proliferationsrate, EBV-DNA $\geq 6,1 \times 10^{7}$ Kopien/ml) und Stadium II 
durch eine Kombination der Bestrahlung mit einer Platin-basierten Chemotherapie Gesamtüberlebensraten (OS) von $78-86 \%$ erreicht werden können [45]. Für die Kombination der Bestrahlung mit einer Chemotherapie stehen entsprechende Therapieprotokolle (2/3 DeVIC: Dexamethason, Etoposid, Ifosfamid und Carboplatin) zur Verfügung [46, 47]. In den fortgeschrittenen Stadien der extranodalen NK/T-Zell Lymphome vom nasalen Typ sind die Ergebnisse der Systemtherapie durch CHOP mit 5-Jahres Überlebensraten um 20\% unbefriedigend, so dass CHOP bzw. Anthrazyklin-haltige Schemata nicht mehr zur Anwendung kommen sollten und durch Protokolle wie SMILE (Asparaginase, Methotrexat, Ifosfamid, Dexamethason und Etoposid) [46, 47] oder VIPD (Etoposid, Ifosfamid, Cisplatin und Dexamethason) [48] zur Anwendung kommen sollten. Desweiteren können die Ergebnisse durch den Einsatz einer konsolidierenden ASCT oder allo SCT deutlich verbessert werden [47, 49].

\subsubsection{Anaplastisches großzelliges Lymphom (ALCL)}

Das Vorliegen des ALK-Fusionsproteins (ALK+) besitzt beim ALCL hohe prognostische Bedeutung, da die 5-Jahres Überlebensrate des ALCL, ALK+ deutlich über der des ALK-negativen Subtyps liegt (70 vs. 49\%) [50].

Für Patienten mit ALK+ ALCL wurden mit 6 Kursen CHOEP in Studien der DSHNHL bzw. mit ACVBP-basierten Protokollen in Studien der GELA für jüngere Patienten Gesamtüberlebensraten nach 5 Jahren von $90 \%$ erreicht $[33,51]$, so dass bei gutem Therapieansprechen für Patienten mit ALK-positivem ALCL eine konsolidierende Hochdosistherapie in der Primärtherapie nicht notwendig erscheint.

Bei ALK-negativen ALCL ist die Prognose unter CHOP bzw. CHOEP als Primärtherapie ungünstiger, so dass eine konsolidierende Hochdosistherapie und ASCT in Betracht gezogen werden sollte. Im Rahmen einer Phase II Studie der Nordic Lymphoma Group wurde für Patienten mit ALK-negativen ALCL durch eine Therapie mit 6 Kursen CHOEP-14, gefolgt von einer Hochdosistherapie mit BEAM und ASCT eine 5-Jahres Gesamtüberlebensrate von 70 \% erreicht [42].

Aufgrund der hohen Expression von CD30 auf den Lymphomzellen steht für Patienten mit rezidivierten und refraktären ALCLs seit 2012 die Therapie mit dem Anti-CD30 Immunkonjugat Brentuximab Vedotin zur Verfügung. Die Gesamtansprechrate ist bei in der Regel guter Verträglichkeit hoch, so dass diese Substanz auch für ein sog. Bridging bis zur ASZT oder alloSZT sinnvoll eingesetzt werden kann [52]. Ob Brentuximab Vedotin in Kombination mit Cyclophosphamid, Adriamycin und Prednison das Vincristin in der Primärbehandlung des ALCL ersetzen kann, wird derzeit in einer internationalen Phase III Studie 6 (6-8 x CHOP-21 versus 6-8 x CHP plus Brentuximab Vedotin) geprüft.

Da ALCL mit einem Anteil von 15\% aller pädiatrischen Lymphome auch bei Kindern und jungen Erwachsenen auftreten, wurden in der pädiatrischen Onkologie umfangreiche Erfahrungen zur Risiko-adaptierten Therapie des ALCL gesammelt [30], so dass bei entsprechend jungen Patienten das Vorgehen im Rahmen von AYA (adolescent and young adult)-Konzepten interdisziplinär festgelegt werden sollte.

\subsubsection{Rezidivtherapie}

Im Rezidiv stehen für Patienten, die einer intensivierten Chemotherapie zugeführt werden können, Platin-haltige zytostatische Kombinationen wie ICE und DHAP analog zu dem Vorgehen bei den DLBCL zur Verfügung [53, 54, 55]. 
Auf Basis von Phase II Studien sind in den USA, nicht jedoch in Europa die HDAC-Inhibitoren Romidepsin [56, 57] und Belinostat [58], sowie der Inhibitor der Thymidilatsynthese Pralatrexat [59] beim rezidivierten/refraktären PTCL zugelassen. Für ALK+ ALCL zeigte die Anwendung von des ALK-Tyrosinkinase-Inhibitors Crizotinib Wirksamkeit im Rezidiv [60,61]. Weitere Studien sind aktiviert. Allerdings kann es nach Absetzen der Medikation zu Wiederauftreten der Erkrankung kommen [62]. Mit diesen Substanzen vergleichbare Ansprechraten von $25-30 \%$ wiesen in kleinen Phase-II-Studien und Fallserien auch Gemcitabin [63], Bendamustin [64] und Lenalidomid [65, 66] auf.

Unabhängig von der gewählten Zweitlinientherapie hat diese für den Großteil der Patienten lediglich palliativen Charakter, ein langfristiges Überleben kann in aller Regel nur durch eine konsolidierende Stammzelltransplantation erzielt werden [54]. Insbesondere durch die alloSZT wurden in mehreren retrospektiven $[67,68,69,70,71,72]$ und prospektiven Studien $[73,74]$ für Patienten mit rezidivierter bzw. refraktärer Erkrankung 3-Jahres Gesamtüberlebensraten von $40 \%$ bis $60 \%$ erreicht, so dass diese Option für geeignete Patienten mit rezidiviertem oder refraktärem PTCL unbedingt in Betracht gezogen werden sollte.

\subsection{Besondere Situationen}

\subsubsection{Enteropathie-assoziertes T-Zell Lymphom (EATL)}

Das Enteropathie-assoziierte T-Zell Lymphom (Typ-1) nimmt aufgrund seiner Genese aus vorbestehender Zöliakie und einer unter CHOP-basierter Therapie sehr ungünstigen Prognose eine Sonderrolle ein.22,75,76 Eine im historischen Vergleich deutliche Verbesserung mit 5-Jahres OS von $60 \%(n=26)$ wurde durch ein intensiviertes Protokoll mit Einsatz von einem Kursus CHOP, gefolgt von 3 Kursen IVE/MTX (Ifosfamid, Vincristin, Etoposid und Methotrexat) und anschließender ASCT erreicht.77

\subsubsection{Brustimplantat-assoziiertes ALCL}

Etwa ab 1997 wurde über das Auftreten von ALCL im Weichgewebe der Brust nach Einsetzen von Implantaten berichtet, und unter dem Begriff Brustimplantat-assoziiertes ALCL (BIAALCL) analysiert [78, 79, 80, 81, 82]. Diese Form des ALCL wurde überwiegend in der Kapsel des Implantats oder in perikapsulären Seromen ohne ausgedehnte Weichteilinfiltrationen der Brust gefunden. Die Inzidenz ist mit ca. 1 in 1 Million pro Jahr nach Einsetzen des Brustimplantats niedrig; die überwiegende Zahl der BIA-ALCL war ALK-negativ. Das diagnostische und therapeutische Vorgehen basierend auf retrospektiven Daten, wurde in einer Konsensuskonferenz [83] diskutiert und umfasst bisher die gezielte diagnostische Abklärung (Punktion mit Zytologie, ggf. Biopsie, Bildgebung) bei Auftreten von Seromen $>12$ Monate nach Einsetzen von Implantaten, sowie bei positiver Zytologie die Entfernung des Implantats und der Kapsel mit anschließenden Verlaufskontrollen. Bei Kapsel-überschreitenden Manifestationen ist eine systemische Chemotherapie analog dem Vorgehen bei ALK-negativen ALCL zu erwägen, siehe auch Wissensdatenbank Anaplastische großzellige Lymphome bei Frauen mit Brustimplantaten.

\subsubsection{Lymphom-assoziierte Hämophagozytische Lymphohistiozytose}

Das Syndrom der Hämophagozytischen Lymphohistiozytose (HLH), klinisch gekennzeichnet durch Fieber, Splenomegalie, Zytopenien und mikroskopisch durch den Nachweis einer 
Hämophagozytose charakterisiert, wird durch eine schwere immunologische Dysregulation verursacht und kann neben einer familiären primären Form bei pädiatrischen Patienten auch bei Erwachsenen sekundär, assoziiert mit Infekten, Autoimmunerkrankungen oder Tumorerkrankungen auftreten. Es wurde hierzu eine amerikanische Website für Ärzte und Betroffene eingerichtet [84]. Die häufigste Ursache der sekundären HLH bei Erwachsenen sind Tumorerkrankungen, insbesondere aggressive Lymphome und unter diesen die PTCL wie das gamma/delta-T-Zell Lymphom $[85,86,87]$. In der diagnostischen Abklärung eines HLH bei einem erwachsenen Patienten sollte daher ein Lymphom, insbesondere ein PTCL, in Betracht gezogen werden. Ausführliche Empfehlungen zu Diagnostik und Therapie finden sich unter Onkopedia Hämophagozytische Lymphohistiozytose (HLH).

\section{Rehabilitation}

Die Rehabilitation nach Therapie der PTCL folgt den Standards, die für die aggressiven B-Zell Lymphome etabliert sind.

\section{Verlaufskontrolle und Nachsorge}

\subsection{Verlaufskontrolle}

Üblicherweise erfolgt die Kontrolle des Therapieerfolges ca. 6 - 8 Wochen nach Therapieende mittels konventioneller CT. In Analogie zu anderen aggressiven Lymphomen ist auch ein PET/ CT möglich, allerdings fehlt hierfür die gesetzliche Grundlage, da diese Untersuchung in Deutschland im Leistungskatalog der Krankenversicherung für Non-Hodgkin Lymphom-Patienten nicht enthalten ist. Im follow-up wird eine bildgebende Diagnostik mittel CT nur bei klinisch symptomatischen Patienten empfohlen, klinische Kontrollen sind alle 3 Monate im ersten Jahr, alle 6 Monate im zweiten Jahr, danach mindestens jährlich empfohlen. 88

Bei den extranodalen NK/T-Zell Lymphomen vom nasalen Typ liegt regelhaft eine EBV-Reaktivierung mit mittels PCR im Blut messbarer Zahl von Virusgenomen vor.89,90 Die EBV-PCR ist als Verlaufsparameter des Therapieansprechens nutzbar.91 Ansonsten folgen die Evaluation des Therapieansprechens und die Nachsorge den Standards, die für die aggressiven BZell Lymphome etabliert sind.

\section{Literatur}

1. Swerdlow SH, Campo E, Harris NL et al.: WHO Classification of Tumours of Haematopoietic and Lymphoid Tissues. Lyon: IARC; 2008

2. De Leval L, Parrens M, Le Bras F et al.: Angioimmunoblastic T-cell lymphoma is the most common T-cell lymphoma in two distinct French information data sets. Haematologica 100:e361-364, 2015. DOI:10.3324/haematol.2015.126300

3. Rüdiger T, Weisenburger DD, Anderson JR et al.: Peripheral T-cell lymphoma (excluding anaplastic large-cell lymphoma): results from the Non-Hodgkin's Lymphoma Classification Project. Ann Oncol 13:140-149, 2002. PMID:11863096

4. Vose J, Armitage J, Weisenburger D: International peripheral T-cell and natural killer/Tcell lymphoma study: pathology findings and clinical outcomes. J Clin Oncol 26:4124-4130, 2008. DOI:10.1200/JCO.2008.16.4558 
5. Jaffe ES: Pathobiology of peripheral T-cell lymphomas. Hematology Am Soc Hematol Educ Program 2006:317-322, 2006. PMID:17124078

6. Wang SS and Vose J: Epidemiology and Prognosis of T-Cell Lymphoma. In T-Cell Lymphomas Foss F. eds. Springer ISBN: 978-1-62703-169-1. 2013

7. Petrich AM, Helenowski IB, Bryan LJ et al.: Factors predicting survival in peripheral Tcell lymphoma in the USA: a population-based analysis of 8802 patients in the modern era. Br J Haematol 168:708-718, 2015. DOI:10.1111/bjh.13202

8. Odejide $\mathrm{O}$, Weigert $\mathrm{O}$, Lane $\mathrm{AA}$ et al.: A targeted mutational landscape of angioimmunoblastic T-cell lymphoma. Blood 123:1293-1296, 2014. DOI:10.1182/ blood-2013-10-531509

9. Cairns RA, Iqbal J, Lemonnier $F$ et al.: IDH2 mutations are frequent in angioimmunoblastic T-cell lymphoma. Blood 119:1901-1903, 2012. DOI:10.1182/ blood-2011-11-391748

10. Couronne L, Bastard C, Bernard OA: TET2 and DNMT3A mutations in human T-cell lymphoma. N Engl J Med 366:95-96, 2012. DOI:10.1056/NEJMc1111708

11. Palomero $\mathrm{T}$, Couronne $\mathrm{L}, \mathrm{Khiabanian} \mathrm{H}$ et al.: Recurrent mutations in epigenetic regulators, RHOA and FYN kinase in peripheral T cell lymphomas. Nat Genet 46:166-170, 2014. DOI:10.1038/ng.2873

12. Sakata-Yanagimoto $M$ : Multistep tumorigenesis in peripheral $T$ cell lymphoma. Int J Hematol 102:523-527, 2015. DOI:10.1007/s12185-015-1738-8

13. Piccaluga PP, Fuligni F, De Leo A et al.: Molecular profiling improves classification and prognostication of nodal peripheral T-cell lymphomas: results of a phase III diagnostic accuracy study. J Clin Oncol 31:3019-3025, 2013. DOI:10.1200/JCO.2012.42.5611

14. Amin HM, Lai R: Pathobiology of ALK+ anaplastic large-cell lymphoma. Blood 110:2259-2267, 2007. PMID:17519389

15. Parrilla Castellar ER, Jaffe ES, Said JW et al.: ALK-negative anaplastic large cell lymphoma is a genetically heterogeneous disease with widely disparate clinical outcomes. Blood 124:1473-1480, 2014. DOI:10.1182/blood-2014-04-571091

16. Went P, Agostinelli C, Gallamini A et al.: Marker expression in peripheral T-cell Iymphoma: a proposed clinical-pathologic prognostic score. J Clin Oncol 24:2472-2479, 2006. PMID:16636342

17. Karube K, Aoki R, Nomura $Y$ et al.: Usefulness of flow cytometry for differential diagnosis of precursor and peripheral T-cell and NK-cell lymphomas: analysis of 490 cases. Pathol Int. 58:89-97, 2008. DOI:10.1111/j.1440-1827.2007.02195.x

18. Horwitz SM, Advani RH, Bartlett NL et al.: Objective responses in relapsed T-cell lymphomas with single-agent brentuximab vedotin. Blood 123:3095-3100, 2014. DOI: 10.1182/blood-2013-12-542142

19. Bossard C, Dobay MP, Parrens M, et al.: Immunohistochemistry as a valuable tool to assess CD30 expression in peripheral T-cell lymphomas: high correlation with mRNA levels. Blood 124:2983-2986, 2014. DOI:10.1182/blood-2014-07-584953

20. Weidmann E, Hinz T, Klein S, et al.: Cytotoxic hepatosplenic gammadelta T-cell lymphoma following acute myeloid leukemia bearing two distinct gamma chains of the T-cell receptor. Biologic and clinical features. Haematologica 85:1024-1031, 2000. PMID:11025592 
21. Chott A, Haedicke W, Mosberger I et al.: Most CD56+ intestinal lymphomas are CD8+CD5-T-cell lymphomas of monomorphic small to medium size histology. Am J Pathol 153:1483-1490, 1998. PMID:9811340

22. Di Sabatino A, Biagi F, Gobbi PG, Corazza GR: How I treat enteropathy-associated T-cell lymphoma. Blood 119:2458-2468, 2012. DOI:10.1182/blood-2011-10-385559

23. Skibola CF, Bracci PM, Nieters A et al.: Tumor necrosis factor (TNF) and lymphotoxinalpha (LTA) polymorphisms and risk of non-Hodgkin lymphoma in the InterLymph Consortium. Am J Epidemiol 171:267-276, 2010. DOI:10.1093/aje/kwp383

24. Manns A, Cleghorn FR, Falk RT et al.: Role of HTLV-I in development of non-Hodgkin lymphoma in Jamaica and Trinidad and Tobago. The HTLV Lymphoma Study Group. Lancet 1993;342:1447-1450, 1993. PMID:7902480

25. Cheung MM, Chan JK, Lau WH et al.: Primary non-Hodgkin's lymphoma of the nose and nasopharynx: clinical features, tumor immunophenotype, and treatment outcome in 113 patients. J Clin Oncol 16:70-77, 1998. PMID:9440725

26. Rizvi MA, Evens AM, Tallman MS et al.: T-cell non-Hodgkin lymphoma. Blood 107:1255-1264, 2006. PMID:16210342

27. Bal M, Gujral S, Gandhi J et al.: Angioimmunoblastic T-Cell lymphoma: a critical analysis of clinical, morphologic and immunophenotypic features. Indian J Pathol Microbiol 53:640-645, 2010. DOI:10.4103/0377-4929.72010

28. Herrera AF, Crosby-Thompson A, Friedberg JW et al.: Comparison of referring and final pathology for patients with T-cell lymphoma in the National Comprehensive Cancer Network. Cancer 120:1993-1999, 2014. DOI:10.1002/cncr.28676

29. Gaulard P, de Leval L: Pathology of peripheral T-cell lymphomas: where do we stand? Semin Hematol 51:5-16, 2014. DOI:10.1053/j.seminhematol.2013.11.003

30. Mussolin L, Damm-Welk C, Pillon $M$ et al.: Use of minimal disseminated disease and immunity to NPM-ALK antigen to stratify ALK-positive ALCL patients with different prognosis. Leukemia 27:416-422, 2013. DOI:10.1038/leu.2012.205

31. Casulo C, Schoder H, Feeney J et al.: 18F-fluorodeoxyglucose positron emission tomography in the staging and prognosis of T cell lymphoma. Leuk Lymphoma 54:2163-2167, 2013. DOI:10.3109/10428194.2013.767901

32. Cottereau AS, Becker S, Broussais F et al. Prognostic value of baseline total metabolic tumor volume (TMTV0) measured on FDG-PET/CT in patients with peripheral T-cell lymphoma (PTCL). Ann Oncol 27:719-724, 2016. DOI:10.1093/annonc/mdw011

33. Schmitz N, Trümper L, Ziepert M et al.: Treatment and prognosis of mature T-cell and NK-cell lymphoma: an analysis of patients with T-cell lymphoma treated in studies of the German High-Grade Non-Hodgkin Lymphoma Study Group. Blood 116:3418-3425, 2010. DOI:10.1182/blood-2010-02-270785

34. Gallamini A, Stelitano C, Calvi R et al.: Peripheral T-cell lymphoma unspecified (PTCL$U)$ : a new prognostic model from a retrospective multicentric clinical study. Blood 103:2474-2479, 2004. PMID:14645001

35. Federico $M$, Rüdiger $T$, Bellei $M$ et al.: Clinicopathologic characteristics of angioimmunoblastic T-cell lymphoma: analysis of the international peripheral T-cell lymphoma project. J Clin Oncol 31:240-246, 2013. DOI:10.1200/JCO.2011.37.3647

36. Voss $\mathrm{MH}$, Lunning MA, Maragulia JC et al.: Intensive induction chemotherapy followed by early high-dose therapy and hematopoietic stem cell transplantation results in 
improved outcome for patients with hepatosplenic T-cell lymphoma: a single institution experience. Clin Lymphoma Myeloma Leuk 13:8-14, 2013. DOI:10.1016/j.clml. 2012.09.002

37. Chalmers AW, Katz DA, Miller IJ, Gregory SA: Successful treatment of hepatosplenic Tcell lymphoma with ESHAP followed by autologous stem cell transplant. Clin Adv Hematol Oncol 11:109-113, 2013. PMID:23598914

38. Ellin $\mathrm{F}$, Landstrom J, Jerkeman M, Relander T: Real-world data on prognostic factors and treatment in peripheral T-cell lymphomas: a study from the Swedish Lymphoma Registry. Blood124:1570-1577, 2014. DOI:10.1182/blood-2014-04-573089

39. Pfreundschuh $M$, Trümper $L$, Kloess $M$ et al.: Two-weekly or 3-weekly $C H O P$ chemotherapy with or without etoposide for the treatment of elderly patients with aggressive lymphomas: results of the NHL-B2 trial of the DSHNHL. Blood 104:634-641, 2004. PMID:15016643

40. Reimer P: Impact of autologous and allogeneic stem cell transplantation in peripheral T-cell lymphomas. Adv Hematol. 2010;2010:320624. DOI:10.1155/2010/320624

41. Reimer $P$, Rüdiger $T$, Geissinger $E$ et al.: Autologous stem-cell transplantation as firstline therapy in peripheral T-cell lymphomas: results of a prospective multicenter study. J Clin Oncol 27:106-113, 2009. DOI:10.1200/JCO.2008.17.4870

42. d'Amore F, Relander T, Lauritzsen GF et al. : Up-front autologous stem-cell transplantation in peripheral T-cell lymphoma: NLG-T-01. J Clin Oncol 30:3093-3099, 2012. DOI: 10.1200/JCO.2011.40.2719

43. Schmitz N, Altmann B, Ziepert M et al. : Allogeneic or autologous transplantation as first-line therapy for younger patients with peripheral T-cell lymphoma: Results of the interim analysis of the AATT trial. J Clin Oncol 2015;suppl; abstr 8507, 2015. http:// meetinglibrary.asco.org/content/149754-156

44. Yang Y, Zhu Y, Cao JZ et al. Risk-adapted therapy for early-stage extranodal nasal-type NK/T-cell lymphoma: analysis from a multicenter study. Blood 126:1424-1432, 2015. DOI:10.1182/blood-2015-04-639336

45. Suzuki R: Pathogenesis and treatment of extranodal natural killer/T-cell lymphoma. Semin Hematol 51:42-51, 2014. DOI:10.1053/j.seminhematol.2013.11.007

46. Yamaguchi M, Kwong YL, Kim WS et al.: Phase II study of SMILE chemotherapy for newly diagnosed stage IV, relapsed, or refractory extranodal natural killer (NK)/T-cell lymphoma, nasal type: the NK-Cell Tumor Study Group study. J Clin Oncol 29:4410-4416, 2011. DOI:10.1200/JCO.2011.35.6287

47. Yamaguchi $M$, Tobinai $K$, Oguchi $M$ et al.: Concurrent chemoradiotherapy for localized nasal natural killer/T-cell lymphoma: an updated analysis of the Japan clinical oncology group study JCOG0211. J Clin Oncol 30:4044-4046, 2012. DOI:10.1200/JCO. 2012.45.6541

48. Kim SJ, Kim K, Kim BS et al.: Phase II trial of concurrent radiation and weekly cisplatin followed by VIPD chemotherapy in newly diagnosed, stage IE to IIE, nasal, extranodal NK/T-Cell Lymphoma: Consortium for Improving Survival of Lymphoma study. J Clin Oncol 27:6027-6032, 2009. DOI:10.1200/JCO.2009.23.8592

49. Jaccard A, Gachard N, Marin B et al.: Efficacy of L-asparaginase with methotrexate and dexamethasone (AspaMetDex regimen) in patients with refractory or relapsing extran- 
odal NK/T-cell lymphoma, a phase 2 study. Blood 117:1834-1839, 2011. DOI:10.1182/ blood-2010-09-307454

50. Savage KJ, Harris NL, Vose JM et al.: ALK- anaplastic large-cell lymphoma is clinically and immunophenotypically different from both ALK+ ALCL and peripheral T-cell lymphoma, not otherwise specified: report from the International Peripheral T-Cell Lymphoma Project. Blood. 2008;111(12):5496-5504, 2008. DOI:10.1182/ blood-2008-01-134270

51. Sibon D, Fournier M, Briere J et al.: Long-term outcome of adults with systemic anaplastic large-cell lymphoma treated within the Groupe d'Etude des Lymphomes de I'Adulte trials. J Clin Oncol 30:3939-3946, 2012. DOI:10.1200/JCO.2012.42.2345

52. Pro B, Advani R, Brice $P$ et al.: Brentuximab vedotin (SGN-35) in patients with relapsed or refractory systemic anaplastic large-cell lymphoma: results of a phase II study. J Clin Oncol 30:2190-2196, 2012. DOI:10.1200/JCO.2011.38.0402

53. Kim MK, Kim S, Lee SS et al.: High-dose chemotherapy and autologous stem cell transplantation for peripheral T-cell lymphoma: complete response at transplant predicts survival. Ann Hematol 86:435-442, 2007. PMID:17256144

54. Mak V, Hamm J, Chhanabhai $M$ et al.: Survival of patients with peripheral T-cell lymphoma after first relapse or progression: spectrum of disease and rare long-term survivors. J Clin Oncol 31:1970-1976, 2013. DOI:10.1200/JCO.2012.44.7524

55. Mikesch JH, Kuhlmann $M$, Demant $A$, et al.: DexaBEAM versus ICE salvage regimen prior to autologous transplantation for relapsed or refractory aggressive peripheral $T$ cell lymphoma: a retrospective evaluation of parallel patient cohorts of one center. Ann Hematol 92:1041-1048, 2013. DOI:10.1007/s00277-013-1738-9

56. Coiffier B, Pro B, Prince HM et al.: Results from a pivotal, open-label, phase II study of romidepsin in relapsed or refractory peripheral T-cell lymphoma after prior systemic therapy. J Clin Oncol 30:631-636, 2012. DOI:10.1200/JCO.2011.37.4223

57. Piekarz RL, Frye R, Prince HM, et al.: Phase 2 trial of romidepsin in patients with peripheral T-cell lymphoma. Blood 117:5827-5834, 2011. DOI:10.1182/ blood-2010-10-312603

58. Zain JM, O'Connor OA, Zinzani PL et al.: Multicenter, open-label trial of PXD 101 in patients with relapsed/refractory peripheral T-cell lymphoma. J Clin Oncol. 2010;suppl; abstr e18565. http://meetinglibrary.asco.org/content/52728-74

59. O'Connor OA, Pro B, Pinter-Brown $L$ et al.: Pralatrexate in patients with relapsed or refractory peripheral T-cell lymphoma: results from the pivotal PROPEL study. J Clin Oncol 29:1182-1189, 2011. DOI:10.1200/JCO.2010.29.9024

60. Gambacorti-Passerini C, Messa C, Pogliani EM : Crizotinib in anaplastic large-cell lymphoma. N Engl J Med 364:775-776, 2011. DOI:10.1056/NEJMc1013224

61. Mosse YP, Lim MS, Voss SD et al.: Safety and activity of crizotinib for paediatric patients with refractory solid tumours or anaplastic large-cell lymphoma: a Children's Oncology Group phase 1 consortium study. Lancet Oncol 14:472-480, 2013. DOI: 10.1016/S1470-2045(13)70095-0

62. Gambacorti-Passerini C, Mussolin L, Brugieres L: Abrupt relapse of ALK-positive lymphoma after discontinuation of crizotinib. N Engl J Med 374:95-96, 2016. DOI: 10.1056/NEJMc1511045 
63. Arkenau HT, Chong G, Cunningham D et al.: Gemcitabine, cisplatin and methylprednisolone for the treatment of patients with peripheral T-cell lymphoma: the Royal Marsden Hospital experience. Haematologica 92:271-272, 2007. PMID:17296587

64. Damaj G, Gressin R, Bouabdallah K et al.: Results from a prospective, open-label, phase II trial of bendamustine in refractory or relapsed T-cell lymphomas: the BENTLY trial. J Clin Oncol 31:104-110, 2013. DOI:10.1200/JCO.2012.43.7285

65. Hopfinger $G$, Nosslinger $T$, Lang $A$ et al.: Lenalidomide in combination with vorinostat and dexamethasone for the treatment of relapsed/refractory peripheral $\mathrm{T}$ cell lymphoma (PTCL): report of a phase I/II trial. Ann Hematol 93:459-462, 2014. DOI: 10.1007/s00277-014-2009-0

66. Morschhauser F, Fitoussi O, Haioun $C$ et al.: A phase 2, multicentre, single-arm, openlabel study to evaluate the safety and efficacy of single-agent lenalidomide (Revlimid) in subjects with relapsed or refractory peripheral T-cell non-Hodgkin lymphoma: the EXPECT trial. Eur J Cancer 49:2869-2876, 2013. DOI:10.1016/j.ejca.2013.04.029

67. Dodero A, Spina F, Narni $F$ et al.: Allogeneic transplantation following a reduced-intensity conditioning regimen in relapsed/refractory peripheral T-cell lymphomas: longterm remissions and response to donor lymphocyte infusions support the role of a graft-versus-lymphoma effect. Leukemia 26:520-526, 2012. DOI:10.1038/leu.2011.240

68. Jacobsen ED, Kim HT, Ho VT et al.: A large single-center experience with allogeneic stem-cell transplantation for peripheral T-cell non-Hodgkin lymphoma and advanced mycosis fungoides/Sezary syndrome. Ann Oncol 22:1608-1613, 2011. DOI:10.1093/ annonc/mdq698

69. Kanakry JA, Kasamon YL, Gocke CD, et al. Outcomes of related donor HLA-identical or HLA-haploidentical allogeneic blood or marrow transplantation for peripheral $\mathrm{T}$ cell lymphoma. Biol Blood Marrow Transplant 19:602-606, 2013. DOI:10.1016/j.bbmt. 2013.01.006

70. Kyriakou C, Canals C, Finke J, et al.: Allogeneic stem cell transplantation is able to induce long-term remissions in angioimmunoblastic T-cell lymphoma: a retrospective study from the lymphoma working party of the European group for blood and marrow transplantation. J Clin Oncol 27:3951-3958, 2009. DOI:10.1200/JCO.2008.20.4628

71. Le Gouill S, Milpied N, Buzyn A, et al.: Graft-versus-lymphoma effect for aggressive Tcell lymphomas in adults: a study by the Societe Francaise de Greffe de Moelle et de Therapie Cellulaire. J Clin Oncol 26:2264-2271, 2008. DOI:10.1200/JCO.2007.14.1366

72. Smith SM, Burns LJ, van Besien K et al.: Hematopoietic cell transplantation for systemic mature T-cell non-Hodgkin lymphoma. J Clin Oncol 31:3100-3109, 2013. DOI:10.1200/ JCO.2012.46.0188

73. Corradini P, Dodero A, Zallio F et al.: Graft-versus-lymphoma effect in relapsed peripheral T-cell non-Hodgkin's lymphomas after reduced-intensity conditioning followed by allogeneic transplantation of hematopoietic cells. J Clin Oncol 22:2172-2176, 2004. PMID:15169805

74. Wulf GG, Hasenkamp J, Jung W et al.: Reduced intensity conditioning and allogeneic stem cell transplantation after salvage therapy integrating alemtuzumab for patients with relapsed peripheral T-cell non-Hodgkin's lymphoma. Bone Marrow Transplant 36:271-273, 2005. PMID:15937499

75. Nijeboer P, Malamut G, Mulder CJ et al.: Enteropathy-associated T-cell lymphoma: improving treatment strategies. Dig Dis 33:231-235, 2015. DOI:10.1159/000369542 
76. Sieniawski MK, Lennard AL: Enteropathy-associated T-cell lymphoma: epidemiology, clinical features, and current treatment strategies. Curr Hematol Malig Rep 6:231-240, 2011. DOI:10.1007/s11899-011-0097-7

77. Sieniawski M, Angamuthu N, Boyd K et al.: Evaluation of enteropathy-associated T-cell lymphoma comparing standard therapies with a novel regimen including autologous stem cell transplantation. Blood 115:3664-3670, 2010. DOI:10.1182/ blood-2009-07-231324

78. Brody GS, Deapen D, Taylor CR et al. Anaplastic large cell lymphoma occurring in women with breast implants: analysis of 173 cases. Plast Reconstr Surg 135:695-705, 2015. DOI:10.1097/PRS.0000000000001033

79. Gidengil CA, Predmore Z, Mattke $S$ et al.: Breast implant-associated anaplastic large cell Iymphoma: a systematic review. Plast Reconstr Surg 135:713-720, 2015. DOI: 10.1097/PRS.0000000000001037

80. Keech JA, Jr., Creech BJ: Anaplastic T-cell lymphoma in proximity to a saline-filled breast implant. Plast Reconstr Surg 100:554-555, 1997. PMID:9252643

81. Miranda RN, Aladily TN, Prince HM et al. Breast implant-associated anaplastic large-cell lymphoma: long-term follow-up of 60 patients. J Clin Oncol 32:114-120, 2014. DOI: 10.1200/JCO.2013.52.7911

82. Anaplastic large cell lymphoma $(A L C L)$ in women with breast implants: Preliminary FDA findings and analyses. http://www.fda.gov/medicaldevices/productsandmedicalprocedures/implantsandprosthetics/breastimplants/ucm239996.htm (Accessed January 2011).

83. Kim B, Predmore ZS, Mattke S et al.: Breast Implant-associated Anaplastic Large Cell Lymphoma: Updated Results from a Structured Expert Consultation Process. Plast Reconstr Surg Glob Open 3:e296, 2015. DOI:10.1097/GOX.0000000000000268

84. Hemophagocytic Syndromes. http://www.histio.org/hemophagocyticsyndromes (Accessed March 2016).

85. Parikh SA, Kapoor $P$, Letendre $L$ et al.: Prognostic factors and outcomes of adults with hemophagocytic lymphohistiocytosis. Mayo Clin Proc 89:484-492, 2014. DOI:10.1016/ j.mayocp.2013.12.012

86. Riviere S, Galicier L, Coppo P et al. Reactive hemophagocytic syndrome in adults: a retrospective analysis of 162 patients. Am J Med 127:1118-1125, 2014. DOI:10.1016/ j.amjmed.2014.04.034

87. Schram AM, Berliner N: How I treat hemophagocytic lymphohistiocytosis in the adult patient. Blood 125:2908-2914, 2015. DOI:10.1182/blood-2015-01-551622

88. d'Amore F, Gaulard P, Trümper L et al. Peripheral T-cell lymphomas: ESMO Clinical Practice Guidelines for diagnosis, treatment and follow-up. Ann Oncol 26 Suppl 5:v108-115, 2015. DOI:10.1093/annonc/mdv201

89. Kwong $Y L$, Pang AW, Leung AY et al.: Quantification of circulating Epstein-Barr virus DNA in NK/T-cell lymphoma treated with the SMILE protocol: diagnostic and prognostic significance. Leukemia 28:865-870, 2014. DOI:10.1038/leu.2013.212

90. Wang L, Wang $\mathrm{H}$, Wang $\mathrm{JH}$ et al. Post-treatment plasma EBV-DNA positivity predicts early relapse and poor prognosis for patients with extranodal NK/T cell lymphoma in the era of asparaginase. Oncotarget 6:30317-30326, 2015. DOI:10.18632/oncotarget. 4505 
91. Au WY, Kim SJ, Yiu HH et al.: Clinicopathological features and outcome of late relapses of natural killer cell lymphomas 10-29 years after initial remission. Am J Hematol 85:362-363, 2010. DOI:10.1002/ajh.21663

\section{Links}

Kompetenznetz maligne Lymphome (KML):

http://www.lymphome.de/

http://www.haarzell-leukaemie.de

\section{Anschriften der Experten}

Dr. med. Georg Hopfinger

Medizinische Universität Wien

Univ. Klinik für Innere Medizin I

Abt. für Knochenmarktransplantation

Währinger Gürtel 18-20

1090 Wien

georg.hopfinger@meduniwien.ac.at

Dr. med. Fatime Krasniqi

Kaderärztin med. Onkologie

Universitätsspital Basel

Petersgraben 4

4031 Basel

Fatime.Krasniqi@usb.ch

Prof. Dr. med. Peter Reimer

Kliniken Essen Süd

Ev. Krankenhaus Essen-Werden gGmbH

Pattbergstr. 1-3

45239 Essen

Tel: 0201 4089-2231

Fax: 0201 4089-2297

p.reimer@evk-werden.de

Prof. Dr. med. Norbert Schmitz

ASKLEPIOS Klinik Hamburg-St. Georg

Hämatologie u. Stammzelltransplantation

Lohmühlenstr. 5

20099 Hamburg

Tel: 040 181885-2005

Fax: 040 188085-4226

n.schmitz@asklepios.com 
Prof. Dr. Gerald Wulf

Universitätsklinikum Göttingen

Abteilung Hämatologie/Onkologie

Robert-Koch-Str. 40

37075 Göttingen

Tel: $055139-6303$

gwulf@med.uni-goettingen.de

\section{Angaben zu möglichen Interessenkonflikten}

nach den Regeln der DGHO Deutsche Gesellschaft für Hämatologie und Medizinische Onkologie und den Empfehlungen der AWMF (Version vom 23. April 2010) und internationalen Empfehlungen

\begin{tabular}{|l|l|l|l|l|l|l|l|}
\hline Name & $\begin{array}{l}\text { Anstellung / } \\
\text { Führungsposi- } \\
\text { tion }\end{array}$ & $\begin{array}{l}\text { Beratung / } \\
\text { Gutachten }\end{array}$ & $\begin{array}{l}\text { Aktien/ } \\
\text { Fonds }\end{array}$ & $\begin{array}{l}\text { Patent / } \\
\text { Urheber- } \\
\text { recht/ } \\
\text { Lizenz }\end{array}$ & $\begin{array}{l}\text { Hono- } \\
\text { rare }\end{array}$ & $\begin{array}{l}\text { Finanzierung wis- } \\
\text { senschaftlicher } \\
\text { Untersuchungen }\end{array}$ & $\begin{array}{l}\text { Andere } \\
\text { finanzielle } \\
\text { Beziehungen }\end{array}$ \\
\hline $\begin{array}{l}\text { Hopfin- } \\
\text { ger }\end{array}$ & $\begin{array}{l}\text { Medizinische } \\
\text { Universität } \\
\text { Wien }\end{array}$ & $\begin{array}{l}\text { Takeda } \\
\text { Pharma- } \\
\text { ceutical }\end{array}$ & - & - & - & - & - \\
\hline Krasniqi & $\begin{array}{l}\text { Universitäts- } \\
\text { spital Basel }\end{array}$ & - & - & - & - & - & - \\
\hline Reimer & $\begin{array}{l}\text { Evangelisches } \\
\text { Krankenhaus } \\
\text { Essen-Werden }\end{array}$ & - & - & - & - & - & - \\
\hline Schmitz & $\begin{array}{l}\text { Asklepios-KIi- } \\
\text { nik St. Georg, } \\
\text { Hamburg }\end{array}$ & - & - & - & - & - & - \\
\hline Wulf & $\begin{array}{l}\text { Universitäts- } \\
\text { medizin Göt- } \\
\text { tingen }\end{array}$ & - & - & - & - & & - \\
\hline
\end{tabular}

